\title{
High-speed railway channel measurements and characterizations: a review
}

\author{
Tao ZHOU*, Cheng TAO, Liu LIU, Jiahui QIU, Rongchen SUN \\ Institute of Broadband Wireless Mobile Communications, Beijing Jiaotong University, Beijing 100044, China
}

\begin{abstract}
Wireless communication for high-speed railways (HSRs) that provides reliable and high data rate communication between the train and trackside networks is a challenging task. It is estimated that the wireless communication traffic could be as high as $65 \mathrm{Mbps}$ per high-speed train. The development of such HSR communications systems and standards requires, in turn, accurate models for the HSR propagation channel. This article provides an overview of existing HSR channel measurement campaigns in recent years. Particularly, some important measurement and modeling results in various HSR scenarios, such as viaduct and U-shaped groove (USG), are briefly described and analyzed. In addition, we review a novel channel sounding method, which can highly improve the measurement efficiency in HSR environment.
\end{abstract}

Key words: high-speed railways; wireless communication; channel measurement; viaduct; U-shaped groove

(C) 2012 JMT. All rights reserved.

\section{Introduction}

$\mathrm{W}$ ith the rapid development of high-speed railways (HSRs), an increasing volume of wireless communication data is required to transfer between the train and the ground. On the one hand, increasing railway controlling information about security monitoring and maintenance needs to be transmitted to the ground; on the other hand, mobile users demand much higher network capacity and more reliable communication quality, independent of their locations or speeds. However, the existing communication system GSM-R (global system for mobile communication for railways) only provides a maximum data rate of less than $200 \mathrm{kbps}$, which is mainly used for railway control and unable to satisfy the requirements of future high speed data transmission. Therefore, a novel broadband wireless communication system is in demand for HSR [1].

The major prerequisite condition of any wideband digital mobile radio system is a thorough knowledge of the propagation characteristics of the mobile radio channel. A reliable and realistic channel model serves as the enabling foundation for practical design and testing of the HSR communication systems. In the actual deployment of a wireless communication system, the actual

Received Oct. 21, 2012; revision accepted Nov. 15, 2012

${ }^{*}$ Corresponding author. E-mail: 11111037@bjtu.edu.cn (T. ZHOU)

(C) 2012 JMT. All rights reserved

doi: 10.3969/j.issn.2095-087X.2012.04.002 network planning optimization, capacity, coverage blind spots, and other works are carried out according to the radio propagation environment. Accurate large-scale channel models, such as path loss (PL) and shadowing fading model, can make the network deployment and the network optimization reliable and efficient, to enhance the performance of the wireless network. Channel smallscale fading features are crucial in physical layer (PHY) design, which help engineers design fading countermeasures, such as diversity of transmission/reception, error correction coding and interleaving, and equalization algorithms.

The features of HSR channels significantly differ from those of mobile cellular communication channels. These differences originate from the following specific features of the HSR radio propagation:

(1) Diverse scenarios. HSR is usually situated on a viaduct or subgrade crossing suburban or rural areas. The transceiver encounters different channel environments, depending on geographic location, viaduct height, antenna position, etc., as shown in Fig. 1. The channel conditions can be classified into several types: viaduct, hilly terrain, U-shaped groove (USG), tunnel, and station. Meanwhile, owing to the high velocity, the train runs across the scenarios so rapidly that a single model can not depict features of HSR channel accurately. Particularly, the propagation characteristics change drastically with the distance between the transceivers even on the same terrain [2]. 
(2) Line of sight (LOS) dominance. The newly-built HSR routes are distinguished from conventional lines, by gentler curves, shallower grooves, and wider tunnels. Combined with high antenna, these external conditions yield a clear or open space for wireless communications. Unlike urban and indoor conditions, in these external conditions, the specular LOS component is much stronger, and other multipath echoes occur less frequently between the mobile terminal and base-station (BS). Accordingly, the channel is generally scattered with a low density, which provides a benign environment for the mobile radio provision.

(3) Large Doppler shift and rapid Doppler transition. The maximum speed of HSR in China is currently $486 \mathrm{~km} / \mathrm{h}$, which induces a Doppler frequency shift of $945 \mathrm{~Hz}$ at $2.1 \mathrm{GHz}$. If the BS is positioned far from the track side, it contributes to a relatively small Doppler shift. The main reason is that the incident angle between the track and the BS will be approximately $90^{\circ}$. However, BSs are often set back $30 \mathrm{~m}$ from the rail. When the train traverses the BS, the angle between the incoming wave and the motion direction will change rapidly from $0^{\circ}$ to $180^{\circ}$, yielding a rapid Doppler transition.

This paper gives an overview of recent studies in HSR channel measurements and models. Then, the existing measurements and results in various HSR scenarios are provided. And then the authors review a highly efficient channel identification method for HSR propagation channels. Finally, current insufficiencies and future work are presented.

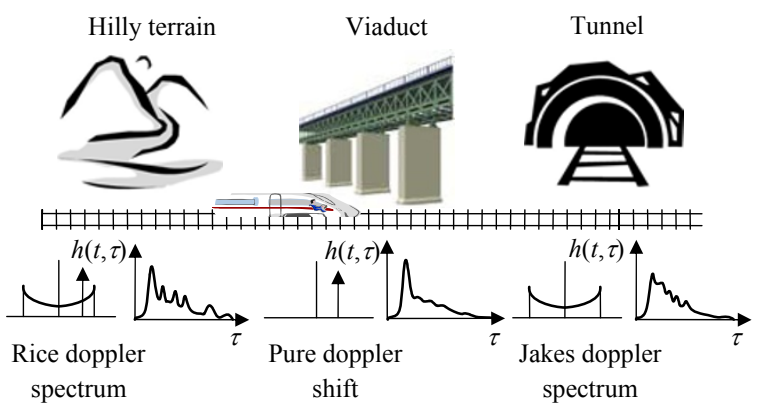

Fig. 1 Different Channel characteristics in different scenarios on HSR [2]

\section{Recent studies in HSR channel measure- ments and characterizations}

\subsection{Existing measurement campaigns}

Most of the previous literature on the channel measurement and characterization is for terrestrial cellular communication systems. However, the underlying HSR channels have not yet been sufficiently investigated. Recently, the important measurements for HSR can be categorized as follows:

(1) Field strength measurements. Field strength was collected on the Nanjing-Qidong line in China at 800 $\mathrm{MHz}$ for the digital trunking system [3]. The measurement covered a group of terrains such as plain, foothill, urban area, and tunnel. The terrain factor influencing prediction of the field strength was obtained. Field strength was gathered in a train-to-train system used for train collision avoidance at $400 \mathrm{MHz}$ [4]. PL, Doppler shift, and delay spread were analyzed in five scenarios (train stations, shunting yards, regional networks, bridges, and tunnels). Field strength measurements were conducted inside a moving train at $320 \mathrm{MHz}$ in western India [5]. In the low-density scattered, rocky, guided and hilly environments, PL models were established to develop mobile-network planning tools. Received signal power was collected on HSR in China at $930 \mathrm{MHz}$ based on the GSM-R [6-7]. PL models in a viaduct and USG scenario were proposed. The fading depth as well as Ricean $K$-factor was parameterized. Furthermore, stationary intervals of time-variant channel are investigated based on the measurement data on the Zhengzhou-Xi'an HSR [8].

(2) Measurements using the special excitation signals. A ray optical deterministic approach to wave propagation modeling in tunnels was presented [9]. Results on channel impulse response (CIR) and the Doppler diagram for links between on-train stations and tunnelfixed stations were provided. RUSK measurement campaign was conducted at $5 \mathrm{GHz}$ in Germany between Siegburg and Frankfurt by Medav, TU-Karlsruhe and TU-Ilmenau. An empirical PL, shadow fading, $K$-factor, and other parameters related to time delay spread as well as the angle spread was presented as rural moving networks called the D2a model in the Winner II model [10]. PropSound measurement was conducted at $2.5 \mathrm{GHz}$ in Taiwan by Elektrobit and the Industrial Technology Research Institute of Taiwan (ITRI) [11]. The propagation parameters investigated were delay spread, maximum excess delay, cluster azimuth spread, global azimuth spread, and temporal behavior of the Doppler power spectrum and angles of arrival/departure. PropSound measurement of the indoor part of HSR train carriage was carried out at the $2.35 \mathrm{GHz}$ band by the China Mobile Research Institute (CMRI) [12]. Based on the omnidirectional ceiling antenna and the planar antenna on the wall, empirical PL model, delay spread, and Ricean $K$-factor for the HSR carriage (relay to terminal) were analyzed and parameterized. PropSound measurement and characterization were conducted at the $2.35 \mathrm{GHz}$ band by Beijing Jiaotong University [2]. A position-based wireless channel model for HSR viaduct scenarios was proposed. 


\subsection{Existing models and standardization}

Multipath models for both dedicated channel (DCH) and high speed downlink packet access (HSDPA) in high-speed train environments were first proposed by Siemens in 2005 [13]. The model is based on the ITU vehicle. The modified model reduces the number of taps and deviates the tap relative mean power. Then, 3GPP defines the HSR model for the test of the baseband performance in TS 25.104 [14], which provides three HSR scenarios, including open space, tunnel with leaky cable, and tunnel for multi-antennas. This model is a nonfading propagation channel model and consists of one tap only. Afterwards, TS 36.104 [15] removes the tunnel propagation scenarios. However, the tunnel scenario cannot be ignored on certain railway lines such as Zhengzhou-Xi'an HSR, because it accounts for $22 \%$ of the whole HSR mileage. Hence, in-depth research on the radio propagation in tunnels is also needed for HSR broadband communication systems. It can be seen that the model in TS 25.104 or TS 36.104 concentrates mainly on the impact of Doppler effect on system performance while ignoring other channel characteristics. WINNER-II and IMT-A channel models [16] contain both large-scale and small-scale properties, which also consider high-speed scenarios in their rural macro-cell (RMa) and moving networks scenarios, respectively. The proposed high-speed scenarios consider dedicated mobile relay stations (MRSs) deployed on the surface of the train to improve the coverage on the carriages by extending the coverage of the outdoor base stations (BSs). Therefore, two types of channels can be distinguished, i.e., BS-MRS and MRS-mobile station (MS) channels. As we know, the existing standardized channel models satisfy the assumption of wide-sense stationary uncorrelated scattering (WSSUS), neglecting the non-stationary of HSR channels. A non-stationary wideband geometrybased stochastic model (GBSM) for multiple-input multiple-output (MIMO) HSR channels is proposed by Ghazal [17]. From this model, the space-time-frequency (STF) correlation function (CF) of different taps is obtained and found to change with time, which proves that the stationary assumption is violated for HST channels. Therefore, non-stationary WINNER/ IMT-A channel model should be investigated in future work.

\section{Measurement results in various HSR scenarios}

\subsection{Measurement system description}

Due to the diverse scenarios on HSR, the existing models discussed in Section 2 cannot characterize the whole HSR channel. Thus, extensive field channel measurement tests in typical scenarios must be carried out. By utilizing the Elektrobit PropSound multidimensional radio channel sounder [18] and the ROHDE \& SCHWARZ TSMQ radio network analyzer, a lot of channel measurements have been performed on BeijingTianjin and Zhengzhou-Xi'an HSR in China, as shown in Table 1. In this section, we address the measurements and results using PropSound.

Table 1 HSR channel measurements

\begin{tabular}{|c|c|c|c|c|c|c|c|c|c|c|}
\hline HSRs & Scenario & $\begin{array}{l}\text { Measurement } \\
\text { mode }\end{array}$ & Equipment & $\begin{array}{l}\text { Excitation } \\
\text { signal }\end{array}$ & $\begin{array}{c}\text { Carrier } \\
\text { frequency } \\
(\mathrm{GHz})\end{array}$ & $\begin{array}{l}\text { Chip } \\
\text { rate }\end{array}$ & $\begin{array}{c}\text { Channel } \\
\text { sampling } \\
\text { frequency }(\mathrm{Hz})\end{array}$ & $\begin{array}{l}\text { Transmit } \\
\text { power } \\
(\mathrm{dBm})\end{array}$ & $\begin{array}{c}\text { Train } \\
\text { velocity } \\
(\mathrm{km} / \mathrm{h})\end{array}$ & $\begin{array}{l}\text { Channel } \\
\text { statistics }\end{array}$ \\
\hline $\begin{array}{l}\text { Beijing- } \\
\text { tianjin }\end{array}$ & Viaduct & $\begin{array}{l}\text { Uplink } \\
\text { SISO }\end{array}$ & PropSound & PN (127) & 2.35 & 5 & 984.25 & 37 & 240 & $\begin{array}{c}\mathrm{PL}, \mathrm{SF}, \\
\mathrm{K}, \mathrm{DF} \\
\mathrm{DS}\end{array}$ \\
\hline $\begin{array}{l}\text { Zhengzhou- } \\
\text { Xi'an }\end{array}$ & Viaduct & $\begin{array}{l}\text { Downlink } \\
\text { SISO }\end{array}$ & PropSound & PN (127) & 2.35 & 25 & 1968.5 & 30.8 & 200 & $\begin{array}{c}\mathrm{PL}, \mathrm{SF}, \\
\mathrm{K}, \mathrm{DF} \\
\mathrm{DS}\end{array}$ \\
\hline $\begin{array}{l}\text { Zhengzhou- } \\
\text { Xi'an }\end{array}$ & USG & $\begin{array}{l}\text { Downlink } \\
\text { SISO }\end{array}$ & PropSound & PN (127) & 2.35 & 25 & 1968.5 & 32.7 & 200 & $\begin{array}{c}\mathrm{PL}, \mathrm{SF}, \\
\mathrm{K}, \mathrm{DF} \\
\mathrm{DS}\end{array}$ \\
\hline $\begin{array}{l}\text { Beijing- } \\
\text { Tianjin, } \\
\text { Zhengzhou- } \\
\text { Xi'an }\end{array}$ & $\begin{array}{l}\text { Viaduct, } \\
\text { USG, } \\
\text { hilly ter- } \\
\text { rain, } \\
\text { plain, } \\
\text { station }\end{array}$ & $\begin{array}{l}\text { Downlink } \\
\text { SISO }\end{array}$ & TSMQ & WCDMA & 2.1 & 3.84 & - & 43 & 240 & DS, K \\
\hline
\end{tabular}

PL: path loss; SF: shadow factor; K: Ricean $K$-factor; DF: Doppler frequency; DS: root mean square (RMS) delay spread 
The measurements were conducted on the No. 0 High Speed Integrated Inspection Train (NHSIIT) that was employed to check the train system administrated by the Ministry of Railways of China. The train-mounted antenna, HUBER+SUHNER [19], was used for transmitting or receiving the radio waves. PropSound utilizes direct sequence spread spectrum signals with the length of 127 to probe the channel and analyze the propagation of radio waves. The receiver records the samples on a hard disk as raw I/Q data. After that, the received signal is correlated with a sequence identical to the one used at the transmitter to recover CIR. Numerous approaches have been developed to determine the noise floor and the threshold decision [20], which can limit the number of taps with non-negligible energy. The clocks at the transmitter and the receiver are synchronized by the GPS network which provides a very accurate universal timing signal to all GPS receivers [21] that also can be used to determine distance.

Large-scale and small-scale characteristics can be obtained by data post-processing. PL is used to predict the signal attenuation as a positive quantity measured in $\mathrm{dB}$, which is defined as the difference between the effective transmitted and the received power. PL typically has a functional relationship according to the distance of the transceiver and is modeled as

$$
L=A+10 n \lg d,
$$

where $L$ is path loss, $d$ is the distance between the transmitter and the receiver in meters, $n$ is the PL exponent, and $A$ is the intercept. Then, the linear regression based on least square (LS) is employed to estimate $A$ and $n$. The shadowing factor can be obtained by subtracting the interpolated PL model from the realistic measurement results. The $K$-factor is the ratio of magnitude of specular components to scatter components in the signal envelope. A moment-based estimator is used to extract the $K$-factor [22]. The Doppler frequency has a significant effect on the average fading rate of the discrete propagation path, which can be observed by the Doppler spectrum. The RMS delay spread, which is the second central moment of power delay profile, describes the dispersion of signals in the time domain.

\subsection{Viaduct scenario in Beijing-Tianjin HSR}

Fig. 2 depicts the measurement scenario and surroundings for Beijing-Tianjin HSR. The Beijing-Tianjin railway scenario is composed of the viaduct and the roadbed, covered with agricultural fields. No scatters and reflectors were observed around the rail. The transmitter equipment was transported by NHSIIT. The receiver was located at Yongledian, which is $80 \mathrm{~km}$ from Tianjin and $60 \mathrm{~km}$ from Beijing. In this campaign, the existing antenna mounted at the roof-top of the carriage is used as the transmitter antenna, about $3 \mathrm{~m}$ above the rail. With a lift truck, a dipole receiver antenna is elevated to approximately $11 \mathrm{~m}$ above the equivalent height of the viaduct, which is positioned approximately $83 \mathrm{~m}$ away from the viaduct rail. Additionally, the viaduct height is $4 \mathrm{~m}$.
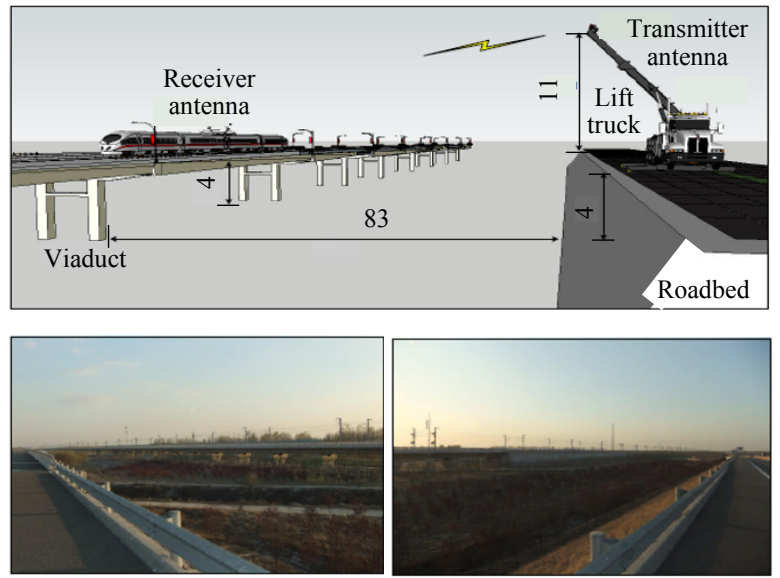

Fig. 2 Propagation condition of Beijing-Tianjin HSR viaduct scenario (unit: $\mathrm{m}$ ) [2]

The measured PL exponent and shadowing factor are 3.03 and $2.0 \mathrm{~dB}$, respectively. The PL exponent is found to be higher than the one in the free space model. This is because the train roof causes distortion in the antenna radiation. The $K$-factor value changes linearly as the train passes through the base-station at distance less than $500 \mathrm{~m}$, whose maximum value reaches approximately $20 \mathrm{~dB}$. However, the $K$-factor value encounters a stable fluctuation in large distances, which can be estimated as a Gaussian variable with a mean value of $8.25 \mathrm{~dB}$ and a standard deviation of $1.05 \mathrm{~dB}$. For the Doppler frequency feature, the phenomenon in TS 25.104 is verified. A specular Doppler frequency shift varies from positive to negative with the angle between the arriving wave and the motion direction. The RMS delay spread, as the train arrives at the remote area, is $0 \mathrm{~ns}$ with the possibility of $50 \%$ due to the existence of a dominant component LOS. The value for the close area lies between $0 \mathrm{~ns}$ and $1000 \mathrm{~ns}$ with the possibility of $90 \%$, which reflects the worst case for frequency selective fading.

\subsection{Viaduct scenario in Zhengzhou-Xi'an HSR}

Fig. 3 shows the measurement scenario and surroundings for Zhengzhou-Xi' an HSR. A vertical-polarized dipole transmitter antenna is positioned on the roof of a farm house at a height of $8 \mathrm{~m}$ above the ground, and elevated to $4.8 \mathrm{~m}$ by a strut bar. This transmitter antenna is approximately $92 \mathrm{~m}$ away from the viaduct rail. The 
receiver antenna is mounted on the top of the train at a height of $3 \mathrm{~m}$ above the rail. The viaduct is surrounded by trees and when the train passes the transmitter nearby, it will encounter the following scenarios: unobstructed, obstructed by tall dense trees, and obstructed by short sparse trees. The tallest trees are about $3 \mathrm{~m}$ higher than the train, and short trees are approximately the same height as the train.

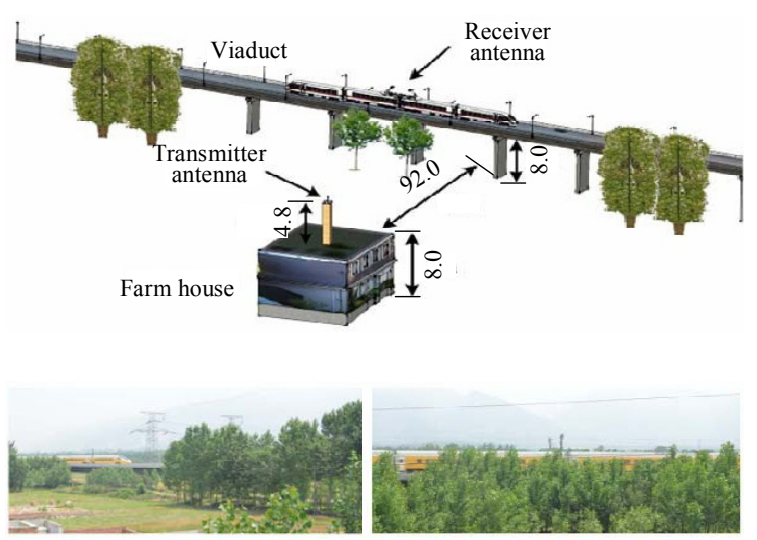

Fig. 3 Propagation condition of Zhengzhou-Xi'an HSR viaduct (unit: $\mathrm{m}$ )

The measurement results are strongly affected by the continuously alternating obstruction of vegetation surrounding the viaduct. The estimated PL exponent is 3.89 , and shadowing factor is $2.5 \mathrm{~dB}$. The $K$-factor varies nonlinearly with the distance. According to the fit to a logistic distribution, the mean value is $2.23 \mathrm{~dB}$ and the standard deviation is $3.79 \mathrm{~dB}$. The Doppler spectrum apparently shows the rapid frequency transition; however, there is also a certain Doppler spread due to the scatter influence of dense trees between the transceivers. In the case of the higher bandwidth of the excitation signal employed in this measurement, cluster delay line (CDL) model is used to characterize the time delay spread. The mean value calculated from all samples of RMS delay spread is $17.38 \mathrm{~ns}$ in the remote area, while the value rises to $72.95 \mathrm{~ns}$ in the close area.

\subsection{U-shaped groove scenario in Zhengzhou-Xi'an HSR}

USG is a special construction of rail with double slope-side walls resulting in a semi-enclosed propagation environment, as shown in Fig. 4. The measured groove is selected near the North of Huashan station, with a depth of about $5 \mathrm{~m}$ and a length of $1000 \mathrm{~m}$. The transmitter antenna is placed on a bridge and elevated to approximately $8.8 \mathrm{~m}$ by a wooden pole, with a small vertical distance to the rail. The receiver antenna is positioned in the middle of the train, mounted on the top with the height of $30 \mathrm{~cm}$ above the roof.
Since the transmitter was positioned close to the railway, the radio propagation was slightly affected by the special structure of USG which may result in a strong reflection and scattering [7]. The PL exponent and shadowing factor were estimated as 2.83 and $1.66 \mathrm{~dB}$, respectively. The $K$-factor can be established as a linear model with a slope of -0.0125 and an intercept of $10.56 \mathrm{~dB}$ as the train travels far from the transmitter in the range of $600 \mathrm{~m}$. If the distance between the transceivers is more than $600 \mathrm{~m}$, the $K$-factor follows a zeromean Guassian distribution with a standard deviation of $5.39 \mathrm{~dB}$. With respect to the Doppler frequency feature, there are just fast transition and little spread in the Doppler spectrum as the train passes through the BS. The RMS delay spread has the possibility of $90 \%$ less than $250 \mathrm{~ns}$. However, when the train is very close to the transmitter, the value decreases to $25 \mathrm{~ns}$, due to the appearance of the strong LOS components.

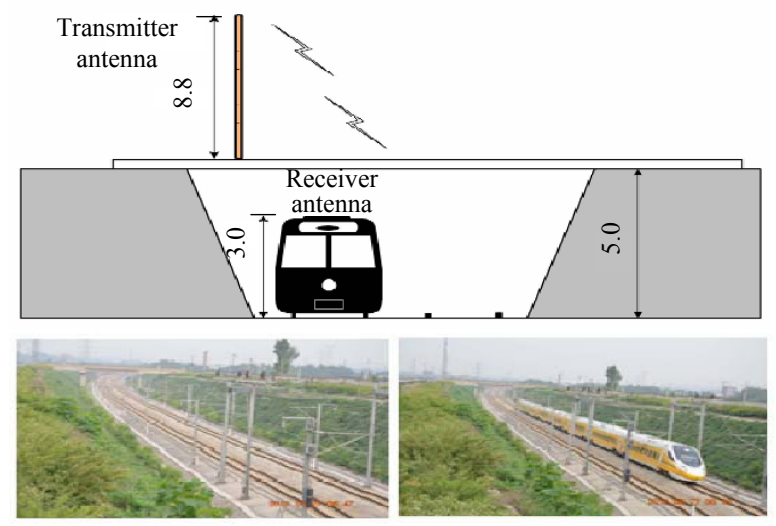

Fig. 4 Propagation condition of USG scenario in Zhengzhou-Xi'an HSR (unit: m)

\section{A highly efficient channel identification method}

In fact, measurement using the dedicated channel sounding equipment like PropSound can just be performed in a certain fixed scenario. This results in low measurement efficiency due to diverse scenarios on HSR. Moreover, since the train travels with a maximum speed of $360 \mathrm{~km} / \mathrm{h}$ and the wireless coverage of a channel sounder is $1 \mathrm{~km}$, the recording time in the coverage is approximately $20 \mathrm{~s}$, which may not be adequate to obtain the statistical property of the time varying channels by the collected data. Hence, a novel efficient channel identification method employing the signal from a wireless cellular system such as wideband code division multiple access (WCDMA) was proposed [23]. This applied signal is treated as the excitation wave, and the stochastic characteristics can be extracted from the received fading components. 
WCDMA is based on the direct-sequence code division multiple access (DS-CDMA) technology and the frequency division duplexing (FDD) mode. The chip rate is $3.84 \mathrm{Mcps}$ with the carrier bandwidth of $5 \mathrm{MHz}$. This wideband signal possesses a large time bandwidth product, low crest factor, and good correlation properties. These merits meet the requirements of a typical excitation signal [24]. In the signal demodulation of the downlink transmission, the time-varying channel information is essentially derived from the common pilot channel (CPICH). CPICH is a broadcast physical channel in cells and does not take any service information. $\mathrm{CPICH}$ is transmitted with a higher power than the dedicated channels and all mobiles in the cell can hear this signal.

The typical WCDMA receiver can combine the received signals from different propagation paths using the Rake receiver. In the system, it aims to collect the channel estimation results before the combination procedure. The channel sounder system components are summarized in the functional block diagram in Fig. 5. After the signal is acquired through the antenna connected to the receiver front end, the received signal is down-converted and subsequently operated by the analogue-digital converter. Then, the time synchronization is performed by the synchronization channel ( $\mathrm{SCH})$. Afterward, the CIR will be derived from the correlator using CPICH. Finally, channel characteristics such as power delay profile, $K$-factor, and Doppler spectrum can be extracted by the CIR.

The TSMQ, accomplishing the synchronization, channel estimation, noise floor selection, and path search, is employed to perform the measurements, as shown in Table 1. Practical experiences reveal that the maximum resolvable time delay is $20 \mu \mathrm{s}$, whereas the minimum resolvable path power is $-20 \mathrm{~dB}$. The RMS delay spread for different regions has been obtained. In the cases of plain and hilly terrains, the RMS delay spread has a similar distribution and yield the smallest values of RMS delay spread. This means that the single path takes the leading position in these two areas because more than $84 \%$ of RMS is zero. The largest PDF

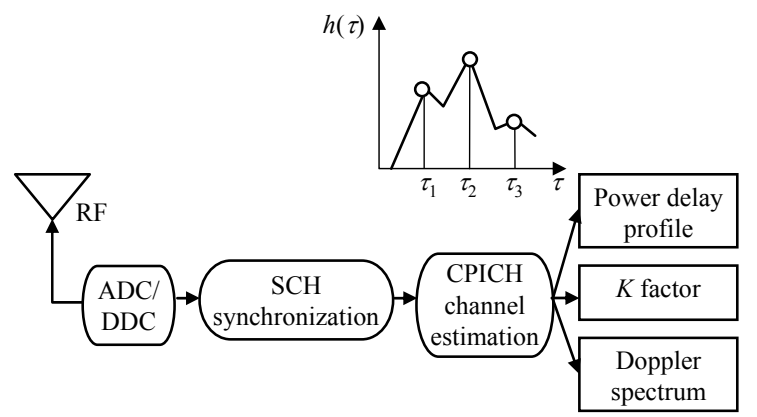

Fig. 5 Functional block diagram of WCDMA receiver [24] of RMS delay spread encountered for USG is approximately $60 \%$, and for the station case, it is less than $20 \%$.

\section{Conclusions}

The HSR propagation channel has a strong impact on the coverage, reliability, and real-time capabilities of HSR wireless networks. Thus, it is vital to use accurate measurement-based models to characterize HSR communications channels. In this paper, we described the results from recent wideband channel measurement campaigns which are useful for the emulation and evaluation of wireless communications in typical HSR propagation scenarios. However, real driving environments do not always adequately satisfy the requirements of the existing models. More comprehensive and distinguishing conditions are required to explore. Multipleinput and multiple-output (MIMO) is widely employed in time division long term evolution (TD-LTE). The correlation of the antennas and angular spread in HSR wireless channel is also attractive. It can be found that the LOS component is extremely strong and fewer multipaths occur in realistic environments. Under this condition, MIMO channels may experience non-independent fading which yields loss in the spatial diversity.

\section{Acknowledgements}

This research was supported in part by the National Natural Science Foundations (Nos.61032002 and 61102050), the National Science and Technology Major Project (No. 2011ZX03001-007-01), the Beijing Natural Science Foundation (No. 4122061), and the Fundamental Research Funds for the Central Universities (No. 2012YJS005).

\section{References}

[1] Y.Q. Zhou, Z.G. Pan, J.L. Hu, et al., Broadband wireless communications on high speed trains, In: Proc. of 20th Annual Wireless and Optical Communications Conf., Newark, NJ, 2011: 1-6.

[2] L. Liu, C. Tao, J. Qiu, et al., Position-based modeling for wireless channel on high speed railway under a viaduct at $2.35 \mathrm{GHz}$, Sel. Area. Comm., 2012, 30(4): 834-845.

[3] Y. Li, Wireless Channel Model Analysis for GSM-R System, [Dissertation], Chengdu: Southwest Jiaotong University, 2007.

[4] C. Garcia, A. Lehner, T. Strang, et al., Channel model for train to train communication using the $400 \mathrm{MHz}$ band, In: Proc. of IEEE Vehicular Technology Conf. 2008, Singapore, May 2008: 3082-3086.

[5] M. Prasad, K. Ratnamla, P. Dalela, Mobile communication measurements along railroads and model evalua- 
tions over Eastern-Indian rural regions, IEEE Antennas. Propag., 2010, 52(5): 131-141.

[6] R. He, Z. Zhong, B. Ai, et al., An empirical path loss model and fading analysis for high-speed railway viaduct scenarios, IEEE Antenn. Wirel. Pr., 2011, 10: 808812.

[7] R. He, Z. Zhong, B. Ai, et al., Propagation measurements and analysis for high-speed railway cutting scenario, Electronics Letters, 2011, 47(21): 1167-1168.

[8] B. Chen, Z. Zhong, B. Ai, Stationary intervals of timevariant channels in high speed railway scenario, China Communications, 2012, 9(8): 64-70.

[9] D. Cichon, T. Zwick, W. Wiesbeck, Radio link simulations in high-speed railway tunnels, In: Proc. of Ninth International Conference on Antennas and Propagation, Eindhoven, Apr. 4-7, 1995: 216-219.

[10] K. Pekka, WINNER II Channel Models part II Radio Channel Measurement and Analysis Results, 2007.

[11] R. Parviainen, K. Pekka, Results of High Speed Train Channel Measurements, in European Cooperation in the Field of Scientific and Technical Research, 2008.

[12] W. Dong, G. Liu, L. Yu, et al., Channel properties of indoor part for high-speed train based on wideband channel measurement, In: Proc. of 5th International ICST Conference on Communications and Networking in China (CHINACOM), 2010, Beijing, Aug. 25-27, 2010: $1-4$.

[13] 3GPP R4-050388, High speed environment channel models, 3GPP TSG-RAN Working Group 4 (Radio) meeting \#35, Athens, Greece, 9th-13th, 2005.

[14] 3GPP Technical Report 25.104, V8.9.0 Release 8, 3rd Generation Partnership Project, Technical Specification Group Radio Access Network, Base Station (BS) radio transmission and reception (FDD), 2009.

[15] 3GPP Technical Report 36.104, V9.3.0 Release 9, Gen- eration Partnership Project, Technical Specification Group Radio Access Network, Evolved Universal Terrestrial Radio Access (E-UTRA), Base Station (BS) radio transmission and reception, 2010.

[16] ITU-R M.2135-1, Guidelines for Evaluation of Radio Interface Technologies for IMT-Advanced, Geneva, Switzerland, Rep. ITU-R M.2135-1, Dec. 2009.

[17] A. Ghazal, C.X. Wang, H. Haas, et al., A non-stationary mimo channel model for high-speed train communication systems, In: Proc. of the 75th Vehicular Technology Conference, Yokohama, Japan, May 6-9, 2012: 1-5.

[18] Elektrobit, PropSoundTM CS Multi-Dimensional Channel Sounder General presentation, Finland: Elektrobit, 2005.

[19] HUBER+SUHNER AG RF Industrial, Sencity Rail Antenna: 1399.17.0039, http://www.firstsourceinc.com/Data Sheets/HuberSuhner/23041568.pdf, 2010-12-30.

[20] J. Dabin, A. Haimovich, H. Grebel, A statistical ultrawideband indoor channel model and the effects of antenna directivity on path loss and multipath propagation, IEEE J. Sel. Area. Comm., 2006, 24(4): 752-758.

[21] N. Costa, S. Haykin, Multiple-Input Multiple-Output Channel Models Theory and Practice, Hoboken: John Wiley \& Sons, Inc., 2010: 137-139.

[22] L. Greenstein, D. Michelson, V. Erceg, Moment-method estimation of the Ricean $K$-factor, IEEE Commun. Lett., 1999, 3(6): 175-176

[23] L. Liu, C. Tao, T. Zhou, Y. Zhao, et al., A highly efficient channel sounding method based on cellular communications for high-speed railway scenarios, http://jwcn. eurasipjournals.com/orderreprints/1687-1499-2012-307, 2012-10-03.

[24] A.F. Molisch, Wireless Communications, 2nd ed., Hoboken: John Wiley and Sons Ltd., 2011.

(Editor: Dongju CHEN) 